\section{Relação entre depressão pós-parto e disponibilidade emocional materna}

\author{
The relationship between postpartum depression \\ and maternal emotional availability
}

\begin{abstract}
Postpartum depression is a highly prevalent disorder that can interfere in the mother-infant relationship. This study aims to evaluate the prevalence of postpartum depression in our sample to compare mother-infant interaction in depressed and non-depressed mothers and to assess the relationship between maternal depression, social support, attachment style, and emotional availability. Participants were pregnant women who planned to deliver at the University of São Paulo Hospital between December 2006 and December 2008. Postpartum depression prevalence in our sample was 28\%. No difference was found in emotional availability between depressed and non-depressed mothers. There was a positive correlation between maternal sensitivity and education, and between sensitivity and some dimensions of social support and attachment styles. We conclude that postpartum depression prevalence in our sample was higher than the average international rates, but that depressive symptoms do not impair mother-infant interaction. Maternal sensitivity is affected by socio-cognitive and emotional factors.
\end{abstract}

Postpartum Depression; Mother-Child Relations; Social Support
Vera Regina J. R. M. Fonseca 1,2

Gabriela Andrade da Silva 1

Emma Otta ${ }^{1}$

\section{Introdução}

As condições físicas e psicológicas da mãe no período que antecede e sucede o parto se revestem de grande importância, já que as bases do desenvolvimento infantil se estabelecem nessa mesma época e dependem intimamente do funcionamento da díade. Assim, a depressão pós-parto, uma condição de alta prevalência em várias culturas e diversas áreas geográficas, foi objeto de inúmeros estudos para avaliar seu impacto na interação mãe-bebê 1,2 e no desenvolvimento deste. A revisão dessa vasta literatura revela resultados por vezes contraditórios.

Vários estudos salientam os efeitos deletérios da depressão pós-parto na interação mãe-bebê 3,4 , tais como: comprometimento do afeto positivo, da sintonia afetiva 5 , da regulação de comportamentos hostis, de intrusividade e falta de contingência 6,7. Por outro lado, os estudos europeus do TCS-PND (Transcultural Study of Postnatal Depression) não conseguiram estabelecer correlação estatisticamente significante entre grau de depressão manifesta e prejuízo na interação mãe-bebê. Entretanto, para o grupo de mães deprimidas, maior intrusividade da mãe estava associada a um melhor engajamento da criança e um comportamento materno mais distante se associava a menor vitalidade da criança ${ }^{8}$. Carter et al. 9, usando a Escala de Disponibilidade Emocional, também não encontraram resultados mais baixos nas mães que apresentavam depres- 
são pós-parto do que nas não deprimidas; apenas as mães deprimidas com outras patologias psiquiátricas foram menos sensíveis.

No Brasil, até o momento, foram encontradas algumas revisões bibliográficas a respeito desse tema 10,11,12 e poucos estudos sobre cuidados maternos na presença de depressão pós-parto 11,13,14, havendo carência de mais dados específicos para nosso país.

\section{Fatores de risco e etiologia}

Em grande parte das culturas e nas diversas áreas geográficas, os fatores de risco para o desencadeamento de um quadro depressivo no pós-parto são semelhantes: perda significativa, estresse, episódio depressivo prévio, gravidez indesejada, dificuldades para lidar com o bebê devido ao temperamento deste ou a doenças, conflito marital, baixo apoio social e dificuldades econômicas 15.

Em uma amostra brasileira, Ruschi et al. 16 encontraram os seguintes fatores associados à depressão materna: menor escolaridade, maior número de gestações, maior paridade, maior número de filhos vivos e menor tempo de relacionamento.

Em um cuidadoso estudo sobre características de temperamento do bebê, Murray et al. 17 concluíram que a irritabilidade do recém-nascido e déficits em sua capacidade de controle motor são fatores preditivos de depressão pósparto, sendo independentes de outras variáveis e significativos per se.

\section{Prevalência}

A prevalência de depressão pós-parto é, em média, de $10 \%$ a $15 \%$, com importantes variações culturais nos fatores de risco. Estudos recentes apontam taxas desde $3,6 \%$ na Alemanha 18 até $34,7 \%$ na África do Sul 19 . Trabalhos brasileiros usando a Escala de Depressão Pós-Parto de Edimburgo (EPDE) relataram prevalências de $12 \% 20$, $22,2 \% 21,36,8 \% 22$ e $37,1 \% 23$. Em recente estudo de Ruschi et al. 16, 39,4\% de uma amostra de 292 mulheres foram consideradas deprimidas, com base na EPDE. A variação nas prevalências provavelmente se deve a fatores metodológicos e contextuais.

Considerando a alta prevalência do transtorno em nosso país, a reavaliação desta, associada a um estudo detalhado das interações na díade mãe-bebê, pode trazer relevantes contribuições para área.

\section{Objetivos}

O presente estudo faz parte de um projeto temático (Projeto Ipê) sobre depressão pós-parto, desenvolvido no Instituto de Psicologia (IP) da Universidade de São Paulo (USP) e no Hospital Universitário (HU) da USP. No que toca ao presente artigo, seus objetivos são:

- avaliar a prevalência de sintomas depressivos entre o segundo e quarto mês após o parto; a depressão pós-parto foi avaliada a partir de nove semanas após o nascimento do bebê até quatro meses;

- investigar a qualidade da interação diádica mãe-bebê na presença de depressão materna em comparação com ausência de depressão, utilizando filmagens na sala de parto e em laboratório - investigar as relações entre a qualidade da interação mãe-bebê e os dados referentes ao estilo de relacionamento da mãe e ao apoio social;

- investigar as relações entre depressão pósparto e apoio social percebido pela mãe e entre depressão pós-parto e estilo de relacionamento da mãe.

\section{Método}

O projeto propõe-se a seguir uma amostra de díades mãe-bebê desde o último trimestre da gestação até os 36 meses de idade da criança. $\mathrm{O}$ grupo de estudo é constituído pelas mães com depressão pós-parto, e o grupo-controle, por mães sem depressão pós-parto.

O presente trabalho irá focalizar os resultados da filmagem realizada aos quatro meses de vida do bebê.

\section{Participantes}

Entre dezembro de 2006 e dezembro de 2008, foram recrutadas gestantes no atendimento prénatal das Unidades Básicas de Saúde (UBS) da Zona Oeste de São Paulo, tendo sido, então, assinado o termo de consentimento livre e esclarecido e feita a primeira entrevista. Novas entrevistas foram realizadas no segundo dia e entre a $9 \underline{a} \mathrm{e}$ 12a semana após o parto. Foram estabelecidas as idades de 4, 8, 12, 24 e 36 meses da criança para as filmagens seguintes.

O critério de inclusão das participantes foi pretenderem dar à luz no HU/USP, local de origem da pesquisa, sendo este o hospital no qual ocorre a maior parte dos partos das mulheres da região atendida pelas UBSs nas quais foi feito o recrutamento. Posteriormente, foram excluídos os casos de prematuridade ou patologia do recém-nascido. 
Instrumentos

\section{- Escala de Depressão Pós-Parto de Edimburgo}

A EDPE 24 é uma escala de autopreenchimento que tem por finalidade a identificação e mensuração da intensidade dos sintomas de depressão pós-parto. O instrumento é composto por dez itens, que recebem pontuação de zero a três, de acordo com a intensidade relatada do sintoma depressivo. A pontuação total na escala varia de 0 a 30, sendo consideradas deprimidas as entrevistadas que tiverem pontuação igual ou superior a 12 na escala 25

\section{- Escala de Disponibilidade Emocional}

A Escala de Disponibilidade Emocional 26, que está sendo usada pela primeira vez no Brasil, avalia a interação mãe-bebê, sendo composta por quatro dimensões do comportamento da mãe e duas do comportamento da criança (para o presente estudo, foi usada apenas uma dimensão da criança, a responsividade):

a) sensibilidade ( 1 a 9 pontos) - expressão e recepção adequadas das emoções por parte da mãe e a resposta pronta e flexível às comunicações da criança;

b) estruturação (1 a 5 pontos) - capacidade materna de fornecer estrutura de interação na qual a exploração da criança coexista com sua autonomia;

c) não-intrusividade (1 a 5 pontos) - estar disponível para a criança sem interferir demasiadamente;

d) não-hostilidade (1 a 5 pontos) - comportamento materno disponível, afetivo, paciente e não agressivo;

e) responsividade da criança à mãe ( 1 a 7 pontos) - capacidade da criança de responder às ações da mãe de modo disponível e afetivo.

\section{- Escala de Apoio Social}

A Escala de Apoio Social (EAS), desenvolvida por Sherbourne \& Stewart 27 e traduzida para o português por Chor et al. 28 , avalia a freqüência com que o respondente pode contar com pessoas que o apóiem em diversas situações. É composta por 19 itens, cada um com cinco alternativas, variando de nunca (1) a sempre (5).

A versão brasileira da escala, além da pontuação total, usa três dimensões 29 : apoio material; apoio afetivo e de interação social positiva; apoio emocional e de informação.

\section{- Escala de Apego Adulto Revisada de Collins}

Uma versão em português da Escala de Apego Adulto de Collins (EAAC) 30,31 foi usada para avaliar os estilos de relacionamento da mãe. O questionário consiste em 18 afirmações que os participantes devem avaliar, decidindo o quanto elas correspondem ao que sentem. Há cinco alternativas de resposta, variando de "Não tem nada a ver comigo" a "Tem tudo a ver comigo". Os itens são divididos entre três escalas (proximidade, confiança e ansiedade), cada uma delas composta por outros seis itens. As pontuações nas subescalas podem variar entre 6 e 30 pontos.

\section{Procedimentos}

As gestantes foram recrutadas no terceiro trimestre de gestação, momento em que também foi realizada a primeira entrevista, que incluía, além de questões estruturadas para obtenção de dados sócio-demográficos, a EAS e a EAAC. Entre dois e quatro meses após o parto, as mesmas mulheres foram entrevistadas novamente, aplicando-se oralmente a EDPE, com base na qual foram formados o grupo de estudo (mães com pontuação igual ou superior a 12) e o grupo-controle (mães com pontuação de 0 a 11).

Quatro meses após o parto, as díades mãebebê foram filmadas no Laboratório de Observação do Comportamento do IP/USP. A filmagem compreendeu 14 minutos de interação mãe-bebê com o uso de brinquedos, e, para sua análise, foi usada a Escala de Disponibilidade Emocional. Os codificadores e a pesquisadora principal (V.R.J.R.M.F.) eram cegos quanto ao status depressivo da mãe. Os vídeos foram avaliados por um grupo de codificadores previamente treinados pelo workshop requerido pela escala $\mathrm{e}$ também pela pesquisadora principal. Caso seus escores fossem muito discordantes daqueles do grupo, chegava-se a um consenso após nova avaliação conjunta.

\section{Análise estatística}

Para avaliar a relação entre a depressão pós-parto e as dimensões de disponibilidade emocional (sensibilidade, estruturação, não-intrusividade, não-hostilidade e responsividade), foram usados testes T para amostras independentes. O mesmo teste foi usado para analisar a relação entre fatores de risco (sexo do bebê, ocupação da mãe, morar com companheiro, gestação planejada e desejada) e disponibilidade emocional.

Para aprofundar a investigação a respeito da relação entre sensibilidade e outras variáveis 
as participantes foram divididas em três grupos conforme suas pontuações na Escala de Disponibilidade Emocional, com base em critério clínico: sensibilidade baixa (pontuação menor que $5, \mathrm{n}=$ 18), média (pontuação entre 5 e $6, \mathrm{n}=72$ ) e alta (pontuação acima de $6, \mathrm{n}=48$ ). Essas categorias foram usadas em testes de análise de variância (ANOVA), que investigaram a relação entre sensibilidade e os fatores de risco: idade e escolaridade da mãe, número de filhos e número de pessoas na casa. Outro teste de ANOVA investigou a relação da sensibilidade (dividida nas categorias citadas) e as subescalas de apego adulto.

Testes de correlação de Pearson foram usados para verificar se havia associação entre as subescalas de disponibilidade emocional entre si e com outras variáveis (pontuações na EAAC e na EAS)

\section{Resultados}

A amostra na qual foi aplicada a EDPE entre a 9a e a 12a semana pós-parto foi de 261 mães. A amostra na qual foi avaliada a disponibilidade emocional, quatro meses após o parto, foi constituída por 138 díades mãe-bebê, sendo 54 com bebês de sexo masculino e 84 com bebês de sexo feminino. A diferença entre as duas amostras decorreu da desistência de algumas participantes ou da impossibilidade de convocá-las por mudança de endereço.

As mães que compuseram a amostra final tinham idade entre 16 e 38 anos (média $=25,3 \pm 5,7$ anos) e haviam estudado entre 1 e 16 anos (média $=9,4 \pm 2,6$ anos de estudo). Sem contar o bebê que é foco da presente pesquisa, as participantes tinham de 0 a 4 filhos (média $=0,8 \pm 0,9$ crianças). Quanto à ocupação, 41,3\% das mães trabalhavam e 9,4\% estudavam. A maioria das mães $(73,9 \%)$ morava com o companheiro durante a gestação, e o número de pessoas que moravam com elas na casa variou entre 1 e 18 (média = $4,0 \pm 2,2$ pessoas).

Comparando o perfil das díades que compuseram a amostra final com o das díades que foram perdidas na observação, foram verificadas duas diferenças significativas: na amostra final, houve um número significativamente maior de bebês de sexo feminino (63\%, comparado com $40,8 \%$ nas díades perdidas na observação. $\left.\chi^{2}{ }_{1}=9,659, \mathrm{p}=0,002\right)$ e uma média maior de pessoas vivendo na mesma casa $(4,01$ e 3,45 , respectivamente. $\left.\mathrm{T}_{212}=2,018, \mathrm{p}=0,045\right)$. Nas outras variáveis indicativas do perfil, não houve diferença significativa entre as amostras inicial e final.

\section{Prevalência}

Do total de 261 mães que responderam a EDPE, 73 apresentaram pontuação maior ou igual a 12 , ou seja, $28 \%$ das gestantes tiveram escores indicativos de depressão pós-parto.

Na subamostra de 138 mães que participaram da análise de interação mãe-bebê aos quatro meses, 39 (29,7\%) tiveram pontuação maior que 12 na EDPE, compondo o grupo de estudo (deprimidas), enquanto 92 tiveram pontuação menor que o ponto de corte, fazendo parte do grupo-controle (não deprimidas).

\section{Relação entre disponibilidade emocional e depressão pós-parto}

$\mathrm{O}$ teste $\mathrm{T}$ mostrou que houve um efeito marginalmente significativo da depressão pós-parto sobre a variável estruturação $[\mathrm{T}(62,8)=-1,849$, $\mathrm{p}=0,069]$. A pontuação média das mães do grupo com depressão pós-parto $(3,72)$ foi ligeiramente mais baixa que a das mães do grupo sem depressão pós-parto $(3,99)$. Não foi encontrada associação estatisticamente significativa entre a depressão pós-parto e as demais variáveis de disponibilidade emocional. As médias das mães dos grupos de estudo e controle em cada uma das escalas de disponibilidade emocional estão apresentadas na Tabela 1 .

\section{Relações internas entre as variáveis de disponibilidade emocional para os grupos com e sem depressão pós-parto}

Para ambos os grupos, verificou-se uma correlação interna robusta entre as variáveis de disponibilidade emocional da mãe, assim como entre estas e a responsividade da criança. Não se replicou o resultado do estudo europeu do TCS-PND, no qual, para o grupo de mães deprimidas, maior intrusividade se ligava à maior responsividade da criança; ao contrário, no grupo-controle, não hostilidade e não-intrusividade não apresentaram correlação estatisticamente significativa com responsividade, mas foi encontrada correlação positiva significativa no grupo de estudo (não hostilidade: $r=0,353, p=0,027$; não intrusividade: $0,382, \mathrm{p}=0,016$ ).

\section{Relação entre disponibilidade emocional e outras variáveis}

Não foi encontrada relação estatisticamente significativa entre as médias nas escalas de disponibilidade emocional e sexo do bebê, ocupação da mãe, residir com o companheiro, planejamento da gestação e ter desejado o bebê. 
Média e desvio-padrão das pontuações nas escalas de disponibilidade emocional no grupo de estudo $(n=39)$ e no grupo controle $(n=92)$.

\begin{tabular}{|c|c|c|c|}
\hline \multicolumn{4}{|l|}{ Emocional } \\
\hline \multicolumn{4}{|l|}{ Sensibilidade (1 a 9) } \\
\hline Média & 5,74 & 6,03 & 0,19 \\
\hline Desvio-padrão & 1,17 & 1,13 & \\
\hline \multicolumn{4}{|l|}{ Estruturação (1 a 5) } \\
\hline Média & 3,72 & 0,77 & 0,05 \\
\hline Desvio-padrão & 3,98 & 0,66 & \\
\hline \multicolumn{4}{|l|}{ Não-intrusividade (1 a 5) } \\
\hline Média & 3,96 & 0,71 & 0,74 \\
\hline Desvio-padrão & 4,01 & 0,79 & \\
\hline \multicolumn{4}{|l|}{ Não-hostilidade (1 a 5) } \\
\hline Média & 4,69 & 0,47 & 0,11 \\
\hline Desvio-padrão & 4,82 & 0,39 & \\
\hline \multicolumn{4}{|l|}{ Responsividade (1 a 7) } \\
\hline Média & 5,00 & 1,24 & 0,54 \\
\hline Desvio-padrão & 5,14 & 1,18 & \\
\hline
\end{tabular}

Apenas três mães disseram, no terceiro trimestre de gestação, que não haviam aceitado a criança até o momento da entrevista. Embora o número seja pequeno para a realização de análise estatística, as médias de disponibilidade emocional das mães que não aceitaram o bebê foram visivelmente menores quando comparadas às médias das outras mães, sobretudo em sensibilidade (média de sensibilidade das mães que aceitaram o bebê: 6,0; média das que não aceitaram: $3,8)$. No entanto, a responsividade dos bebês foi, em média, semelhante nos dois grupos.

Testes de ANOVA foram feitos para investigar se a sensibilidade, dividida nas categorias baixa (menor que 5), média (5 a 6) e alta (maior que 6), apresentava associação com a idade, escolaridade e número de filhos da participante, bem como com o número de pessoas que moravam em sua residência.

Foi encontrada diferença estatisticamente significativa entre os grupos para a variável anos de escolaridade $\left(\mathrm{F}_{2,119}=7,339, \mathrm{p}<0,001\right)$. O posthoc Tukey's B mostrou que a diferença nas médias se deu no grupo de sensibilidade baixa (média = 7,20 ), cujas mães haviam estudado menos que as dos grupos de sensibilidade média (média $=9,31$ ) e alta (média $=9,90$ ).

\section{Relação entre disponibilidade emocional e variáveis referentes ao estilo de relacionamento da mãe}

Houve correlação significativa entre as pontuações das mães nas subescalas de proximidade e de confiança da EAAC e nas subescalas de sensibilidade e estruturação da disponibilidade emocional, conforme mostra a Tabela 2. Também houve correlação positiva significativa entre pontuação na EDPE e ansiedade, e correlação negativa entre essa mesma pontuação e os itens proximidade e confiança.

Pontuações mais altas em proximidade correlacionaram-se com pontuações mais altas em sensibilidade $(r=0,252, p=0,009)$ e estruturação $(0,311, p=0,001)$. O mesmo ocorreu com a subescala confiança: pontuações mais altas nessa escala estiveram significativamente correlacionadas com sensibilidade $(\mathrm{r}=0,250, \mathrm{p}=0,010) \mathrm{e}$ estruturação ( $r=0,256, p=0,008)$.

Uma ANOVA mostrou que a sensibilidade da mãe, quando transformada nas categorias baixa, média ou alta, evidenciou diferenças significativas quanto à subescala proximidade $[\mathrm{F}(2)=7,521$, $\mathrm{p}=0,001]$. O post-hoc Tukey's B mostrou que as mães de sensibilidade baixa (média $=19,31$ ) apresentaram média significativamente inferior à apresentada pelas mães das categorias média (média $=22,76)$ e alta (média $=23,89$ ). Não foi 
Correlações entre as pontuações nas subescalas da Escala de Apego Adulto Revisada de Collins e pontuações nas dimensões da Escala de Disponibilidade Emocional e na Escala de Depressão Pós-Parto de Edimburgo (EPDE).

\begin{tabular}{lccc}
\hline & Ansiedade & Proximidade & Confiança \\
\hline Pontuação EDPE & $0,401 *$ & $-0,228 * \star$ & $-0,500 *$ \\
Sensibilidade & 0,016 & $0,252 * \star *$ & $0,250 * \star \star$ \\
Estruturação & $-0,046$ & $0,311 * \star *$ & $0,256 * \star$ \\
Não-intrusividade & 0,026 & 0,115 & 0,145 \\
Não-hostilidade & 0,050 & 0,130 & 0,183 \\
Responsividade & 0,124 & 0,109 & 0,138 \\
\hline
\end{tabular}

* $\mathrm{p}<0,001$;

** $p<0,05$;

$\star \star \star * ~ p<0,01$.

encontrado efeito significativo de sensibilidade sobre outras subescalas de apego adulto.

\section{Apoio social e disponibilidade emocional}

Testes de correlação de Pearson foram realizados para investigar a associação entre o apoio social na gestação e (i) a pontuação das mães na EDPE, aplicada de dois a quatro meses após o parto, e (ii) as pontuações de disponibilidade emocional da díade quatro meses após o parto. Os resultados podem ser observados na Tabela 3 .

Houve correlação negativa estatisticamente significativa entre a pontuação na EDPE e a pontuação total na EAS, bem como com suas dimensões emocional e de informação e afetivo e de interação positiva. Esse resultado indica que o apoio social durante a gestação agiu de forma protetora, estando associado a sintomas depressivos menos intensos no puerpério.

Em relação à disponibilidade emocional, foram encontradas correlações positivas significativas entre apoio social e a estruturação da mãe e também entre apoio social e responsividade do bebê. A não-hostilidade apresentou correlação positiva estatisticamente significante com a dimensão de apoio afetivo e de interação positiva.

\section{Discussão}

Em nossa amostra, encontramos uma prevalência de sintomatologia depressiva bastante superior à média mundial $(10 \%$ a $15 \%)$ e próxima às taxas mais altas referidas para o Brasil (entre $32,9 \%$ e $37,1 \%)$.

A Escala de Disponibilidade Emocional, usada pela primeira vez no Brasil, apresentou cor- relação interna satisfatória, o que pode ser considerado um indicador de que seus critérios são aplicáveis a nossa população.

Entretanto, a qualidade da interação entre a mãe e a criança aos quatro meses, avaliada por essa mesma escala, não foi diferente nos grupos com e sem depressão pós-parto, exceção feita a uma diferença marginal quanto à dimensão estruturação. Podemos considerar, a partir de tais resultados, que a relação mãe-bebê aos quatro meses não seja amplamente afetada pela depressão pós-parto medida pela EPDE, como já foi apontado por Gunning et al. 8. Por outro lado, sendo a disponibilidade emocional uma escala holística, aspectos mais sutis, porém importantes da interação, não são avaliados. Acreditamos que será interessante incluirmos na análise dos vídeos outras dimensões, tais como contingência e sincronia, sorriso ${ }^{32}$ e contato de olhar. Além disso, deve-se levar em conta a possibilidade de que a alta taxa de perda, próxima de $50 \%$, tenha interferido nos resultados (as mães desistentes poderiam, por exemplo, ser aquelas com menor disponibilidade emocional).

Prosseguindo na análise dos resultados, veremos que novamente a estruturação apresenta uma correlação positiva com o apoio social percebido pela mãe: quanto mais a mãe se sente amparada afetiva e materialmente pelo ambiente social, mais ela é capaz de organizar a atividade da criança e prover regras e estímulos de acordo com a necessidade individual e da faixa etária. Note-se que a responsividade do bebê também apresenta correlação positiva, ainda que fraca, com o apoio emocional, de informação e material percebido pela mãe.

Na mesma linha, a estruturação também caminha junto com as dimensões proximidade e 
Correlações de Pearson entre as pontuações na Escala de Apoio Social na gestação, a pontuação na Escala de Depressão Pós-Parto de Edimburgo (EPDE), entre 2 e 4 meses após o parto, e nas escalas de disponibilidade emocional quatro meses após o parto $(\mathrm{n}=138)$

\begin{tabular}{lcccc}
\hline & $\begin{array}{c}\text { Suporte emocional } \\
\text { e de informação }\end{array}$ & $\begin{array}{c}\text { Suporte afetivo } \\
\text { e de interação }\end{array}$ & $\begin{array}{c}\text { Suporte } \\
\text { material }\end{array}$ & $\begin{array}{c}\text { Suporte } \\
\text { total }\end{array}$ \\
\hline Pontuação EDPE & $-0,366^{*}$ & $-0,466^{*}$ & $-0,203 * *$ & $-0,411 *$ \\
Sensibilidade & 0,111 & 0,154 & 0,090 & 0,138 \\
Estruturação & $0,226 * * *$ & $0,260 * * *$ & $0,180 * *$ & 0,144 \\
Não-intrusividade & 0,114 & 0,103 & $-0,009$ & 0,133 \\
Não-hostilidade & 0,120 & $0,199 *$ & 0,134 \\
Responsividade & $0,195^{*}$ & 0,130 & $0,210 *$ & $0,199 *$ \\
\hline
\end{tabular}

* $\mathrm{p}<0,001$;

** $\mathrm{p}<0,05$;

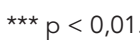

confiança da EAAC. Talvez a dimensão estruturação reflita a capacidade materna de se envolver ativamente com a criança e sua disponibilidade para ir além do atendimento de necessidades afetivas e de cuidado (este último refletido na dimensão sensibilidade), conseguindo ser criativa sem ser intrusiva, incentivando, assim, a responsividade do bebê.

Ao dirigirmos nosso foco para a dimensão sensibilidade, perceberemos uma nítida associação entre ela e escolaridade materna e aceitação da gravidez. Além disso, a EAAC revela que as mães menos sensíveis apresentam tendência de se sentirem menos queridas e de serem mais desconfiadas em suas relações. Tais resultados apontam para o envolvimento de fatores tanto sócio-cognitivos, que são proporcionados pela educação, quanto afetivos e de personalidade, expressos na aceitação ou não da gravidez e nos esquemas relacionais pré-existentes. Por outro lado, o apoio social percebido pela mãe não afeta sua sensibilidade. Convém notar que, na análise dos vídeos, a sensibilidade da mãe associase com frequência à capacidade de interpretar o bebê e fazer hipóteses sobre seus estados, revelando que a criança já é considerada em sua subjetividade.

A questão referente a quais aspectos do cuidado materno e da interação são avaliados pelas várias dimensões do constructo da disponibilidade emocional está em aberto, mas é um campo com importantes implicações para o estudo das relações iniciais entre a mãe e o bebê e o impacto dessas relações no desenvolvimento infantil.

Os resultados do TCS-PND 8, que revelavam uma associação entre maior intrusividade ma- terna e maior responsividade da criança no grupo de mães deprimidas, não foram replicados em nossa amostra. Para o grupo com depressão pós-parto, mães mais sensíveis são também mais estruturantes, menos intrusivas e menos hostis sendo seus bebês mais responsivos. Para o grupo-controle, essas relações se mantêm apenas para sensibilidade e estruturação; neste caso, bebês mais responsivos não têm necessariamente mães menos intrusivas ou menos hostis. Uma hipótese possível seria que bebês de mães não deprimidas seriam mais tolerantes aos comportamentos intrusivos e hostis das mães; os bebês de mães deprimidas seriam responsivos apenas na presença de condições de boa disponibilidade materna.

Para finalizar, é importante ressaltar que o apoio social percebido pela mãe, além de apresentar correlação positiva com a estruturação materna e com a responsividade do bebê, funciona como fator protetor para sintomas depressivos.

Tais resultados apontam para a importância de se considerar a gestação e o puerpério como momentos críticos para o desenvolvimento da criança, merecendo atenção especial dos responsáveis pelas políticas de saúde.

Quanto às limitações do estudo, em primeiro lugar, ao nos referirmos à depressão pós-parto, não estamos falando de diagnóstico de depressão, mas de sintomas depressivos referidos pela própria participante. No entanto, segundo o estudo de validade da EDPE realizado em Brasília a correlação de Spearman entre o diagnóstico pelo Research Diagnostic Criteria (RDC) e a pontuação na escala foi de 0,78 . Usando o ponto de corte $11 / 12$, a escala apresentou sensibilidade 
(proporção de verdadeiro positivo, ou seja, mães que foram consideradas deprimidas pelo RDC e pela EDPE) de $72 \%$ e especificidade (proporção de verdadeiro negativo, ou seja, mães que não foram consideradas deprimidas pelo RDC nem pela EDPE) de $88 \% 25$.

\section{Resumo}

A depressão pós-parto é um transtorno de alta prevalência que pode comprometer a qualidade da relação mãe-criança. Este estudo pretende determinar a prevalência do referido transtorno, comparar a interação mãe-bebê nos grupos com e sem depressão e verificar a relação entre depressão, apoio social e estilos de relacionamento e disponibilidade emocional maternos. As participantes eram gestantes que pretendiam dar à luz no Hospital Universitário da Universidade de São Paulo entre dezembro de 2006 e dezembro de 2008. A prevalência de depressão pós-parto em nossa amostra foi $28 \%$. Não houve diferença significativa na relação mãe-criança no grupo com e sem depressão. Encontrou-se correlação positiva entre sensibilidade materna e escolaridade e entre sensibilidade e certas dimensões de apoio social e estilo de relacionamento. Conclui-se que a prevalência de depressão pós-parto em nossa amostra é mais alta que a média mundial, mas a sintomatologia depressiva não interfere significativamente na qualidade da interação mãe-bebê. A sensibilidade materna é influenciada por fatores sócio-cognitivos e afetivos.

Depressão Pós-Parto; Relações Mãe-Filho; Apoio Social
Em segundo lugar, ainda que tenha havido controle quanto ao fator subjetivo na análise dos vídeos, a subjetividade é inerente à escala usada, com a possibilidade de introdução de vieses.

\section{Colaboradores}

V. R. J. R. M. Fonseca contribuiu com a aplicação do protocolo para avaliação da disponibilidade emocional, treinamento da equipe de codificadores de disponibilidade emocional, análises e discussão dos dados, além de ter redigido o artigo. G. A. Silva contribuiu com a aplicação das escalas (EDPE, apoio social, Collins), análises e discussão dos dados e participou da redação do artigo. E. Otta supervisionou todas as etapas da pesquisa e revisou a versão final do manuscrito.

\section{Agradecimentos}

Nossos agradecimentos aos codificadores: Ana Rita Rocha, Daniel Kauffmann, Denise Serber, Juliana Amaral, M. Ângela Trombetti Moraes, M. Cecília Pereira da Silva, M. Helena Badra Maaz, M. Teresa Ferriani Nogueira, Mariângela Mendes de Almeida e Patrícia Vendramin. O projeto temático sobre depressão pós-parto é financiado pela Fundação de Amparo à Pesquisa do Estado de São Paulo (FAPESP). V. R. J. R. M. Fonseca é apoiada pela FAPESP. G. A. Silva é apoiada pela FAPESP e pela Coordenação de Aperfeiçoamento de Pessoal de Nível Superior (CAPES). E. Otta é apoiada pelo Conselho Nacional de Desenvolvimento Científico e Tecnológico (CNPq). 


\section{Referências}

1. Reck C, Hunt A, Fuchs T, Weiss R, Noon A, Moehler $E$, et al. Interactive regulation of affect in postpartum depressed mothers and their infants: an overview. Psychopathology 2004; 37:272-80.

2. Edhborg M, Lundh W, Seimyr L, Widström A. The parent-child relationship in the context of maternal depressive mood. Arch Womens Ment Health 2003; 6:211-6.

3. Reissland N, Shepherd J, Herrera E. The pitch of maternal voice: a comparison of mothers suffering from depressed mood and non-depressed mothers reading books to their infants. J Child Psychol Psychiatry 2003; 44:255-61.

4. Edhborg M, Lundh W, Seimyr L, Widström A-M. The long-term impact of postnatal depressed mood on mother-child interaction: a preliminary study. J Reprod Infant Psychol 2001; 19:61-71.

5. Stanley C, Murray L, Stein A. The effect of postnatal depression on mother-infant interaction, infant response to the Still-face perturbation, and performance on an Instrumental Learning task. Dev Psychol 2004; 116:1-18.

6. Jacinthe E. Les effets de la dépression maternelle sur la relation mèreenfant au cours des trois premières années de vie: état de larecherche actuelle et analyse critique. Can Psychol 2001; 42:153-72.

7. Cohn J, Campbell S, Matias R, Hopkins J. Faceto-face interactions of postpartum depressed and nondepressed mother-infant pairs at 2 months. Dev Psychol 1990; 26:15-23.

8. Gunning M, Conroy S, Valoriani V, Figueiredo B, Kammerer M, Muzik M, et al. Measurement of mother-infant interactions and the home environment in a European setting: preliminary results from a cross-cultural study. Br J Psychiatry 2004; 184:38-44.

9. Carter AS, Garrity-Rokous FE, Chazan-Cohen R, Little C, Briggs-Gowan MJ. Maternal depression and comorbidity: predicting early parenting, attachment security, and toddler social-emotional problems and competencies. J Am Acad Child Adolesc Psychiatry 2001; 40:18-26.

10. Schmidt E, Piccoloto N, Müller M. Depressão pósparto: fatores de risco e repercussões no desenvolvimento infantil. Psico USF 2005; 10:61-8.

11. Frizzo GB, Piccinini CA. Interação mãe-bebê em contexto de depressão materna: aspectos teóricos e empíricos. Psicol Estud 2005; 10:47-55.

12. Motta MG, Lucion AB, Manfro GG. Efeitos da depressão materna no desenvolvimento neurobiológico e psicológico da criança. Rev Psiquiatr Rio Gd Sul 2005; 27:165-76.

13. Medeiros P. Perfil dos cuidados maternos em mães deprimidas e não deprimidas no período puerperal. J Bras Psiquiatr 2004; 53:227-34.

14. Frizzo G, Piccinini C. Depressão materna e a interação triádica pai-mãe-bebê. Psicol Reflex Crít 2007; 20:351-60.

15. Goldbort J. Transcultural analysis of postpartum depression. MCN Am J Matern Child Nurs 2006; 31:121-6.

16. Ruschi GEC, Sun SY, Mattar R, Chambô Filho A, Zandonade E, Lima VJ. Aspectos epidemiológicos da depressão pós-parto em amostra brasileira. Rev Psiquiatr Rio Gd Sul 2007; 29:274-80.
17. Murray L, Stanley C, Hooper R, King F, Fiori-Cowley A. The role of infant factors in postnatal depression and mother-infant interactions. Dev Med Child Neurol 1996; 38:109-19.

18. Ballestrem C, Strauss M, Kächele H. Contribution to the epidemiology of postnatal depression in Germany - implications for the utilization of treatment. Arch Womens Ment Health 2005; 8:29-35.

19. Cooper P, Tomlinson M, Swartz L, Woolgar M, Murray $\mathrm{L}$, Molteno C. Post-partum depression and the mother-infant relationship in a South African periurban settlement. Br J Psychiatry 1999; 175:554-8.

20. Da Silva VA, Moraes-Santos AR, Carvalho MS, Martins ML, Teixeira NA. Prenatal and postnatal depression among low income Brazilian women. Braz J Med Biol Res 1998; 31:799-804.

21. Zaconeta AM, Motta LD, França PS. Depresión postparto: prevalencia de test de rastreo positivo en puérperas del Hospital Universitário de Brasília, Brasil. Rev Chil Obstet Ginecol 2004; 69:209-13.

22. Skafuzka E. Prevalência da depressão pós-parto e fatores associados em mães que deram à luz em um Hospital Universitário do Município de S. Paulo [Tese de Doutorado]. São Paulo: Faculdade de Medicina, Universidade de São Paulo; 2000.

23. Cruz EBS, Simões GL, Faisal-Cury A. Rastreamento da depressão pós-parto em mulheres atendidas pelo Programa de Saúde da Família. Rev Bras Ginecol Obstet 2005; 27:181-8.

24. Cox JL, Holden JM, Sagovsky R. Detection of postnatal depression: development of the 10-item Edinburgh Postnatal Depression Scale. Br J Psychiatry 1987; 150:782-6.

25. Santos MFS. Depressão no pós-parto: validação da Escala de Edimburgo em puérperas brasilienses [Dissertação de Mestrado]. Brasília: Universidade de Brasília; 1995.

26. Biringen Z, Robinson J, Emde R. Appendix B: the emotional availability scales. Attach Hum Dev 2000; 2:256-70.

27. Sherbourne CD, Stewart AL. The MOS Social Support Survey. Soc Sci Med 1991; 32:705-14.

28. Chor D, Griep RH, Lopes CS, Faerstein E. Medidas de rede e apoio social no Estudo Pró-Saúde: pré-testes e estudo piloto. Cad Saúde Pública 2001; 17:887-96.

29. Griep RH, Chor D, Faerstein E, Werneck GL, Lopes CS. Validade de constructo de escala de apoio social do Medical Outcomes Study adaptada para o português no Estudo Pró-Saúde. Cad Saúde Pública 2003; 21:703-14.

30. Collins NL, Read SJ. Adult attachment, working models, and relationship quality in dating couples. J Pers Soc Psychol 1990; 58:644-63.

31. Collins NL. Working models of attachment: implications for explanation, emotion, and behavior. J Pers Soc Psychol 1996; 71:818-32.

32. Otta E. O sorriso e seus significados. Petrópolis: Editora Vozes; 1994.

Recebido em 23/Set/2009

Versão final reapresentada em 16/Jan/2010

Aprovado em 01/Mar/2010 